\title{
The perceived opportunity to avoid pain paradoxically increases pain-related fear through increased threat appraisals
}

Citation for published version (APA):

van Vliet, C. M., Meulders, A., Vancleef, L. M. G., \& Vlaeyen, J. W. S. (2021). The perceived opportunity to avoid pain paradoxically increases pain-related fear through increased threat appraisals. Annals of Behavioral Medicine, 55(3), 216-227. https://doi.org/10.1093/abm/kaaa045

Document status and date:

Published: 01/03/2021

DOI:

10.1093/abm/kaaa045

Document Version:

Publisher's PDF, also known as Version of record

\section{Document license:}

Taverne

Please check the document version of this publication:

- A submitted manuscript is the version of the article upon submission and before peer-review. There can be important differences between the submitted version and the official published version of record.

People interested in the research are advised to contact the author for the final version of the publication, or visit the DOI to the publisher's website.

- The final author version and the galley proof are versions of the publication after peer review.

- The final published version features the final layout of the paper including the volume, issue and page numbers.

Link to publication

\footnotetext{
General rights rights.

- You may freely distribute the URL identifying the publication in the public portal. please follow below link for the End User Agreement:

www.umlib.nl/taverne-license

Take down policy

If you believe that this document breaches copyright please contact us at:

repository@maastrichtuniversity.nl

providing details and we will investigate your claim.
}

Copyright and moral rights for the publications made accessible in the public portal are retained by the authors and/or other copyright owners and it is a condition of accessing publications that users recognise and abide by the legal requirements associated with these

- Users may download and print one copy of any publication from the public portal for the purpose of private study or research.

- You may not further distribute the material or use it for any profit-making activity or commercial gain

If the publication is distributed under the terms of Article $25 \mathrm{fa}$ of the Dutch Copyright Act, indicated by the "Taverne" license above, 


\title{
The Perceived Opportunity to Avoid Pain Paradoxically Increases Pain-Related Fear Through Increased Threat Appraisals
}

\author{
Christine M. van Vliet, MSc ${ }^{1,2, \bullet}$ Ann Meulders, PhD $^{1,2}$ Linda M. G. Vancleef, PhD $^{2}$ Johan W. S. Vlaeyen, PhD $^{1,2}$
}

Published online: 25 July 2020

(C) Society of Behavioral Medicine 2020. All rights reserved. For permissions, please e-mail: journals.permissions@oup.com.

\begin{abstract}
Background Although pain-related avoidance is mainly intended to reduce the accompanying anticipatory fear, avoidance behavior may paradoxically increase fear when a previous avoidance response is no longer available, suggesting that there is a bidirectional relationship between pain-related fear and avoidance.
\end{abstract}

Purpose We hypothesized that avoidance can serve as a source of information that fuels irrational pain-related threat appraisals, which, in turn, increases pain-related fear.

Methods Participants $(N=66)$ were exposed to a painful heat stimulus and randomly assigned to the avoidance or control group. They were instructed to avoid the full heat intensity by pressing a stop button in the presence of a stop cue. Only avoidance group participants received a stop cue and were allowed to press the stop button, while control group participants received the same instructions but never had the opportunity to avoid the full heat intensity. In reality and unknown to participants, the intensity and duration of the heat stimulus was independent of the avoidance response. In the subsequent test phase, the avoidance response was unavailable for both groups. We measured pain-related fear, threat appraisals/harmfulness, and pain intensity.

Results In line with our expectations, pain-related fear levels were higher when the avoidance response was no longer available compared to those when the avoidance response was available. Increased threat appraisals

Christine van Vliet

christine.vanvliet@kuleuven.be

$1 \quad$ Research Group Health Psychology, Faculty of Psychology and Educational Sciences, KU Leuven, Tiensestraat 102, Box 3726, 3000 Leuven, Belgium

2 Experimental Health Psychology, Maastricht University, Maastricht, The Netherlands mediated the relationship between avoidance behavior and increased pain-related fear.

Conclusions The perceived opportunity to avoid increased pain-related fear through threat appraisals, suggesting a more complicated relationship between pain-related fear, threat appraisals, and avoidance behavior than the unidirectional relationships proposed in the fear-avoidance model. Clinical implications are discussed.

Keywords: Avoidance $\cdot$ Pain-related fear $\cdot$ Threat appraisals $\cdot$ Pain intensity

\section{Introduction}

How we experience pain can be influenced by beliefs, expectations, motivations, and emotions, as well as our own behavior [1]. The fear-avoidance model describes two pathways in response to pain initiated by an injury. If a pain experience is interpreted as a sign of (impending) harm, pain-related fear may occur and associated avoidance behavior, which ultimately can lead to disability, disuse, and depression. On the contrary, the nonthreatening appraisal of pain will more likely lead to confrontation and speedy recovery [2-4]. A relatively large body of evidence is in line with this model's assumptions, and a systematic review and meta-analysis concluded that, despite the low quality of the investigations, pain-related fear is associated with chronic musculoskeletal pain and disability and is a precursor to chronic pain and disability [5]. In addition, several studies have demonstrated high levels of pain-related fear to result in avoidance behavior (e.g., [6-7]). However, less is known about how avoidance behavior affects fear and threat appraisals.

The bidirectional relationship between perceived threat and safety-seeking behaviors has been proposed to contribute to the persistence of anxiety disorders [8]. 
Safety-seeking behaviors typically prevent the disconfirmation of imminent threat [9]. More recent anxiety research revealed that engaging in avoidance behavior may bear threat-inducing properties [10, 11]. Van den Hout et al. reported that highly anxious people infer higher threat when they avoid more [12]. Lovibond et al. showed that threat appraisals (such as the expectancy of an unconditioned stimulus; i.e., US expectancy) regulate fear and that avoidance aims to reduce the expectancy of a threatening outcome [9]. In that sense, individuals use their avoidance behavior to infer that the stimulus, which is avoided, might be harmful. For example, because an individual suffering from chronic low-back pain has been avoiding lifting a heavy object, (s)he might consider lifting that object as a harmful movement, although this might not necessarily be the case. In line with this reasoning, preliminary evidence for the hypothesis that pain avoidance increases pain-related fear was provided in our previous study [13]. We reported increased pain-related fear levels for those avoiding a painful heat stimulus compared to those who did not have the opportunity to avoid that stimulus. In our experimental paradigm, avoidance group participants were led to believe that they could avoid the full intensity of the painful heat stimulus by pressing the stop button, while control group participants believed that they were exposed to the maximum heat stimulus at all times. However, the duration and intensity of the heat stimulus were kept equal in both groups and did not change throughout the experiment. Only for the avoidance group, the visual feedback about the rising temperature of the heat stimulus stopped before it reached its maximum upon instructed button press, creating the illusion of avoiding the maximal intensity of the painful heat stimulus.

The observation that fear increased after exerting avoidance behavior provides evidence for the bidirectionality hypothesis. The current study aims to replicate our previous work [13], and extending this to provide more insight into the underlying mechanisms of the bidirectional relationship between pain-related fear and avoidance. Compared to the earlier study, we include more measurement times for assessing fear, pain intensity, and threat appraisal to follow the temporal dynamics and to investigate the mediating role of threat appraisal in the avoidance-fear relationship. We hypothesized the following: (a) the prior possibility to avoid the painful heat stimulus increases pain-related fear (self-reports and eyeblink startle response) of subsequent pain stimuli when the option to avoid is not available anymore; (b) the perceived avoidance attenuates pain reports despite exposure to identical physical stimulus intensity; and (c) the change in threat appraisal of pain mediates the relationship between avoidance behavior and subsequent increase of pain-related fear.

\section{Methods}

\section{Participants}

A total of 66 healthy, pain-free volunteers participated in the study (50 females; mean \pm standard deviation $(S D$; range) age $=22.24 \pm 4.75(18-41)$ years). Participants were recruited at KU Leuven using social media and distribution of flyers around the campus. Psychology students received course credits for participation; other participants received a monetary compensation of $€ 8$. Participants were excluded if they reported to suffer from any cardiovascular disease, chronic pain condition, pain at the nondominant forearm, psychiatric disorder (current or in the past), neurological condition, or to be pregnant. The Social and Societal Ethics Committee of KU Leuven approved the experimental protocol (Reg \#: G-2017-10-960). All participants provided written informed consent, which stated that they were allowed to decline participation at any time during the experiment without any consequences. Participants were randomly assigned, via block randomization, to one of two experimental groups: the avoidance group ( $n=33,29$ females) or the control group ( $n=33,21$ females).

\section{Apparatus}

Phasic painful heat stimuli were generated by a Peltier element-based computer-controlled thermal stimulation device (Medoc, TSA, RAMA Yishau, Israel) and delivered through a thermode surface of $30 \times 30 \mathrm{~mm}^{2}$ attached to the nondominant medial forearm. Acoustic startle probes (white noise delivered at $102 \mathrm{dBA}$ with instantaneous rise time of $50 \mathrm{~ms}$ ) were presented binaurally using headphones (Hoher, Stereo headphones, HF92) to elicit the eyeblink startle responses to measure painrelated fear [14].

\section{Study Protocol}

We used a between-subject design with an avoidance group that was presented with the perceived opportunity to avoid and a control group that never received the opportunity to avoid. The protocol is almost identical to the one reported in our previous study [13], apart from the following methodological improvements: (a) painrelated fear and threat levels are now measured at trial level; (b) although the control group is never given the opportunity to avoid (i.e., they are never presented with the stop cue), instructions to avoid the maximal painful heat stimulus are given to both groups and not just to the avoidance group. The experiment was conducted during one single 60-min session and consisted of the 
following phases: preparation and calibration, habituation, experiential, full intensity, intervention, and test. After the experiment, participants completed a number of questionnaires (see 2.4 Questionnaires). This study was preregistered at Open Science Framework (https:// osf.io/dwt9y).

\section{Experimental task}

Participants observed a red vertical bar filling up on a computer screen during each heat stimulus presentation, providing visual feedback about the progress of the rising temperature of the heat stimulus. The vertical bar depicted the labels "baseline" at the bottom of the bar and "maximum" at the top of the bar (for an overview of the experimental design and trial structure, see Fig. 1). While the temperature was rising, the bar gradually filled up.

Preparation and calibration phase. Participants received oral and written information about the experiment. They were informed that they would be exposed to painful heat stimuli but that the stimulus intensity would be individually determined during the calibration phase. After signing the informed consent, we attached the thermode to the nondominant medial forearm. Next, during the calibration phase, the heat stimulus intensity was set at individual pain threshold $(\mathrm{PTH})$ and $1^{\circ}$ degree above the pain threshold, which was the maximum intensity $\left(\mathrm{PTH}+1{ }^{\circ} \mathrm{C}\right.$; see our previous study for a detailed description of the heat stimulus calibration procedure [13]). Once the individual intensity was determined, the presentation of each heat stimulus throughout the experiment was as follows: the heat stimulus always started at a baseline temperature of $10^{\circ} \mathrm{C}$ below the maximum intensity and ramped up with a rate of $0.5^{\circ} \mathrm{C} / \mathrm{s}$ to the individually determined maximum temperature $\left(\mathrm{PTH}+1{ }^{\circ} \mathrm{C}\right)$ and remained at that temperature level for $5 \mathrm{~s}$.

Habituation phase. This phase is included to establish a reliable baseline level of startle responding. Participants were instructed that they would be presented with "short, loud noises" throughout the experiment. Next, 10 auditory startle probes were presented with a variable intertrial interval of $10-15 \mathrm{~s}$. No painful heat stimuli were given during this phase.

Experiential phase. This phase is included for participants to learn that the visual feedback on the screen actually corresponds to the experienced temperature on the arm. All participants received two trials, during which the heat stimulus reached the maximum PTH + $1{ }^{\circ} \mathrm{C}$ temperature and the visual feedback displayed that the maximum temperature was reached (bar colored red

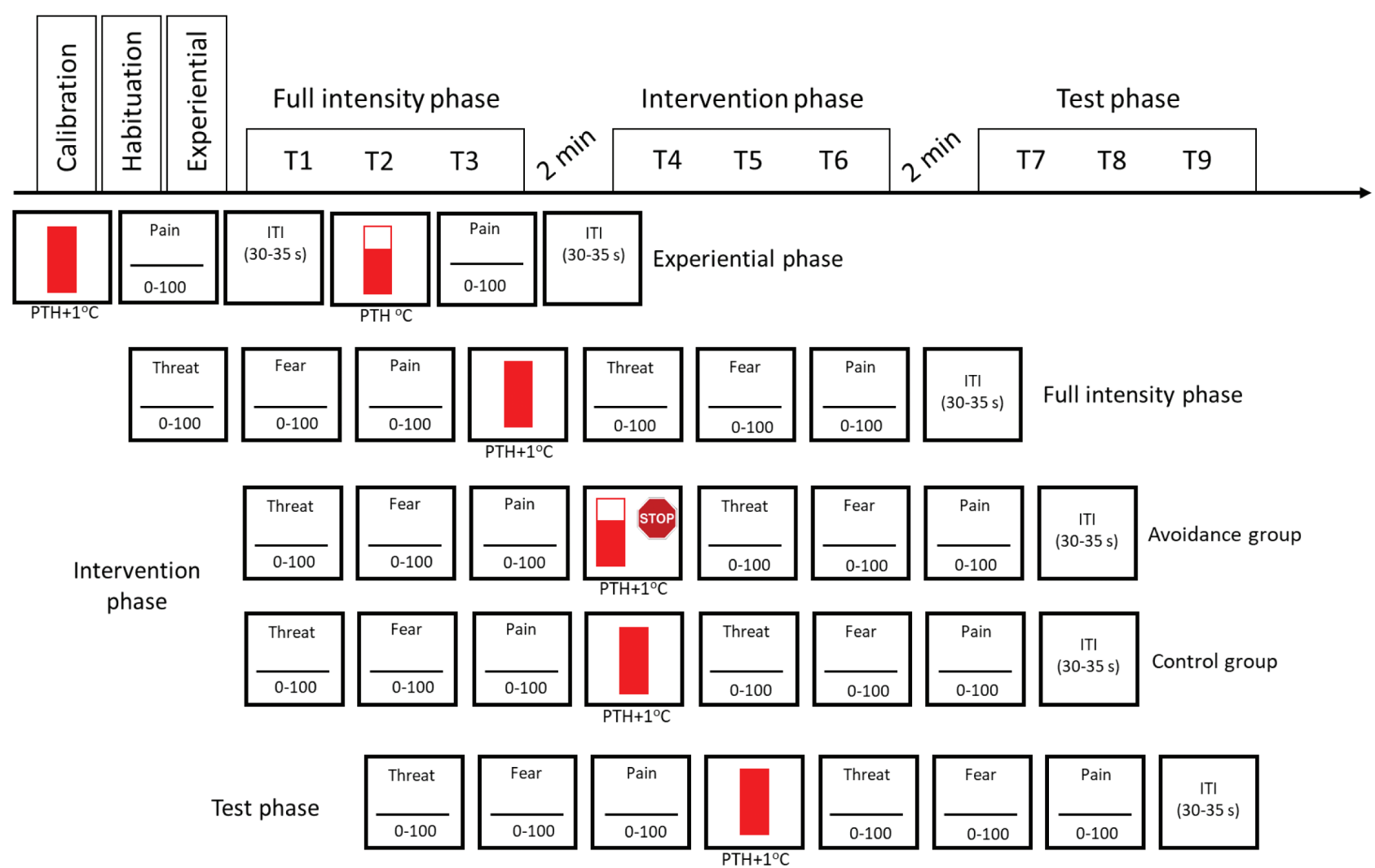

Fig. 1. Overview of the experimental design and exemplary trial structure. $\mathrm{PTH}+1^{\circ} \mathrm{C}=$ maximum temperature; ITI $=$ intertrial interval; calibration $=$ determine individual pain threshold level; habituation $=$ presentation of 10 startle probes. 
until label "maximum"). Next, two trials followed where the heat intensity reached the PTH temperature and the visual feedback stopped before it reached its maximum.

Full-intensity phase. All participants received three trials, during which the heat stimulus and visual feedback concurrently stopped at maximum heat pain intensity $(\mathrm{PTH}$ $+1^{\circ} \mathrm{C}$ ) and, thus, the visual feedback bar filled up until the maximum.

Intervention phase. Both groups received the following instructions: "As soon as you see the stop-cue on the screen, press the stop-button immediately to stop the heat stimulation." The stop cue was a stop sign presented next to the visual feedback bar on the screen. The stop cue always appeared at the same time, very close to the end of the stimulation during the intervention phase for the avoidance group. However, participants in the control group never saw the stop cue during the intervention phase and, thus, were never allowed to perform the avoidance response. As a result, they received three trials during which the visual feedback again stopped at its maximum $\left(\mathrm{PTH}+1{ }^{\circ} \mathrm{C}\right)$. In contrast, avoidance group participants received the stop cue during the intervention phase trials. Upon emitting the avoidance response (i.e., pressing the stop button), the visual feedback stopped before it reached its maximum. In reality, however, unknown to the participants in the avoidance group, the bar filled up at a slower pace, as a result of which the participants received the maximum intensity heat stimulus $\left(\mathrm{PTH}+1{ }^{\circ} \mathrm{C}\right)$ similar to the control group.

Test phase. Both groups received the following instruction: "The stop-cue will no longer be presented, you cannot stop the stimulation anymore." Next, they received three full-intensity heat stimuli in which the visual feedback bar also reached its maximum.

\section{Questionnaires}

For descriptive purposes, participants completed several questionnaires upon completion of the experiment. We measured trait anxiety with the trait version of the StateTrait Anxiety Inventory (STAI-T) [15]; fear of pain with the Dutch version of the Fear of Pain Questionnaire (FPQ-III NL) [16], and positive and negative affect with the Positive Affectivity and Negative Affectivity Scale (PANAS) [17].

\section{Manipulation Check}

At the end of the experiment, two single items on an anchored scale from 0 to 100 assessed the perceived control over the painful heat stimulus ("When it was possible, to what extent did you feel you could influence the duration and thus also the intensity of the heat stimulus?") and the credibility of the visual feedback on the screen, respectively ("To what extent was the visual feedback on the screen accurate about the stimulus intensity?"). These questions served as manipulation checks.

\section{Outcome Measures}

\section{Self-reports}

On a trial-by-trial basis, participants rated pain-related fear, the perceived harmfulness of the stimulus (threat appraisal), and pain intensity before and after each heat stimulation (i.e., prospective/retrospective fear and perceived harmfulness ratings; expected/experienced pain intensity ratings) on a scale from 0 to 100 , with the following questions: (a) "How afraid are/were you of the heat stimulus?"; (b) "How harmful will the heat stimulus be/ was the heat stimulus?"; (c) "How painful will the heat stimulus be/was the heat stimulus?"

\section{Startle eyeblink modulation}

In addition to the self-reports, a psychophysiological correlate of fear (i.e., the eyeblink startle response) was measured (for an overview of psychophysiological correlates of pain-related fear see [18]). The startle reflex, which is triggered by startle-evoking stimuli (in this case, an acoustic probe), is a cross-species, full-body reflex involved in defensive response mobilization. The eyeblink response is one component of the startle response. In human fear conditioning research, eyeblink startle responses are generally measured by recording the surface electromyography (EMG) activity on the M. orbicularis oculi beneath the left eye. Electrodes were attached according to the site specifications described by Blumenthal et al. [14]. The raw signal was amplified by a Coulbourn isolated bioamplifier with a bandpass filter (LabLinc v75-04). The recording bandwidth of the EMG signal was between $90 \mathrm{~Hz}$ and $1 \mathrm{kHz}$. The signal was rectified online and smoothed by a Coulbourn multifunction integrator (LabLinc v763-23A) with a time constant of $20 \mathrm{~ms}$. The EMG signal was digitized at $1,000 \mathrm{~Hz}$ from $500 \mathrm{~ms}$ before the onset of the auditory startle probe until $1,000 \mathrm{~ms}$ after probe onset. Throughout all the experimental phases, startle probes were presented during each trial (trial duration: $26.5 \mathrm{~s}$ ): two during the heat stimulus (an early probe, between 2 and $8 \mathrm{~s}$, and a late probe toward the end of the stimulation, between 18 and $24 \mathrm{~s}$ ), and one startle probe was presented randomly during the intertrial interval (ITI: $30-35 \mathrm{~s}$, between 10 and $20 \mathrm{~s}$ ). We included the probe during ITI as a baseline comparison (participants should have learned that no painful stimulations were given during ITI). Preprocessing occurred as follows: we calculated the peak amplitudes using a Matlab script. Every peak amplitude was defined as the maximum of the response curve within $21-175 \mathrm{~ms}$ 
after probe onset and was scored by subtracting its baseline score (averaged EMG level between 1 and $20 \mathrm{~ms}$ after probe onset). The raw scores were transformed to $\mathrm{T}$-scores to account for interindividual differences in physiological reactivity. All startle waveforms were visually inspected for technical abnormalities and artifacts. All startle data were included during the analysis.

\section{Data Analysis Strategy}

First, descriptive statistics were computed to describe the sample and to test for group differences on the different psychological trait questionnaires. Second, to check whether our manipulation worked, independent samples $t$-tests were conducted to compare the perceived control over the heat stimulus and the credibility ratings of the visual feedback on the screen between the avoidance and control group. Third, to test the first hypothesis that prior possibility to avoid the painful heat stimulus increases pain-related fear of subsequent pain stimuli when the option to avoid is not available anymore, we conducted 2 Group (avoidance and control) $\times 9$ Trial $(\mathrm{T} 1-\mathrm{T} 9)$ repeated-measures $(\mathrm{RM})$ analyses of variance (ANOVAs) on prospective and retrospective pain-related fear ratings. Furthermore, we performed planned comparisons between the last trial of the fullintensity phase and the first trial of the test phase. We expected larger effects of losing the opportunity to avoid on the prospective pain-related fear ratings than on the retrospective pain-related fear ratings. We assume the ratings to be updated after participants experienced the heat stimulus. In other words, participants feel less afraid after they experienced the "maximum" heat stimulus again during test. Additionally, we performed a 2 Group (avoidance and control) $\times 2$ Probe (during stimulation and ITI) $\times 9$ Trial $($ T1-T9) RM ANOVA on the startle data. We carried out planned comparisons between the last trial of the full-intensity phase and the first trial of the test phase. In addition, because responses to a single probe are not always reliable, we performed a 2 Group (avoidance and control) $\times 2$ Probe (during stimulation and ITI) $\times 3$ Phase (full intensity, intervention, and test) RM ANOVA on the startle data to confirm whether similar results emerged as observed in the trial-level analysis. See Supplementary Material for analysis.

Fourth, to test our second hypothesis that perceived avoidance attenuates pain reports despite exposure to identical physical stimulus intensity, we performed a 2 Group (avoidance and control) $\times 9$ Trial $($ T1-T9) RM ANOVA on expected and experienced pain intensity ratings. Planned comparisons were performed on the pain ratings for the last trial of the full-intensity phase versus the last trial of the intervention phase, as well as for the last trial of the intervention phase versus the first trial of the test phase.
For each significant RM ANOVA effect, $\eta \mathrm{g} 2$ is reported as the recommended effect size statistic for RM designs [19]. In case of violation of sphericity, Greenhouse-Geisser corrections were applied. All statistical tests are considered significant at $p<.05$. HolmBonferroni corrections were applied to correct for multiple comparison testing.

Finally, to test our third hypothesis whether threat appraisal (as measured by harmfulness of the painful heat stimulus) mediates the effect of avoidance on pain-related fear, ordinary least-squares path analysis was employed to estimate coefficients in the model [20,21]. This model was tested using regression (to calculate statistics for specific paths) and bootstrapping (to generate a confidence interval $[\mathrm{CI}]$ for the mediation effect/indirect effect). The bootstrapping method is considered the most powerful, most effective method in small samples, and the least vulnerable to Type I errors. In addition, bootstrapping is a nonparametric resampling procedure, which does not assume normal distributions for any variable. We resampled the data 10,000 times. More specifically, we expected that the threat appraisal (measured as the prospective harmfulness of the painful stimulus) increases from the last trial of the intervention phase (Trial 6) to the first trial of test phase (Trial 7) for participants of the avoidance group and that this increase in prospective threat appraisal mediates the increase in prospective pain-related fear. To test the mediation, we performed the following steps: we calculated the increase in perceived harmfulness and in painrelated fear by subtracting the first trial of the test phase by the last trial of the intervention phase, which gave us the variables of interest: $\Delta$ threat and $\Delta$ fear. With these two variables, we performed the mediation analysis as described above. $\Delta$ threat may be considered a mediator to the extent to which it carries an influence of group allocation (avoidance/control group; independent variable) to the $\Delta$ fear (dependent variable). Hence, mediation can be said to occur when there is a significant indirect effect. The test on the indirect effect is based on bootstrap 95\% CIs. If $95 \% \mathrm{CI}$ of the indirect effect does not contain zero, we would say mediation has occurred.

\section{Results}

\section{Descriptive Statistics}

Groups did not differ on self-reported pain intensity or temperature (PTH and $\mathrm{PTH}+1{ }^{\circ} \mathrm{C}$ ) of the stimulation during calibration (on a scale from 0 to 100), indicating that the heat stimuli were perceived similar across groups at the onset of the experiment. There were no significant differences in trait anxiety (STAI-T), fear of pain (FPQIII-NL), and positive and negative affect (PANAS) between groups. Please see Table 1 for an overview. 
Table 1. Descriptive statistics and independent samples $t$-tests for the questionnaires scores for both the avoidance group $(n=33)$ and the control group $(n=33)$ separately

\begin{tabular}{|c|c|c|c|c|}
\hline Total & Avoidance group & Control group & & \\
\hline$N=66$ & $M(S D)$ & $M(S D)$ & $t(64)$ & $p$ \\
\hline Temperature PTH $\left({ }^{\circ} \mathrm{C}\right)$ & $43.55(2.74)$ & $43.70(2.72)$ & 0.22 & .82 \\
\hline Pain intensity PTH & $57.21(19.16)$ & $56.03(18.92)$ & 0.25 & .80 \\
\hline Pain intensity $\mathrm{PTH}+1$ & $72.05(16.19)$ & $71.53(16.03)$ & 0.13 & .89 \\
\hline STAI-T-total & $37.61(8.29)$ & $40.15(8.52)$ & -1.23 & .22 \\
\hline FPQ - total & $75.33(14.15)$ & $77.00(9.46)$ & -0.56 & .58 \\
\hline PANAS - positive & $36.09(4.53)$ & $33.73(5.60)$ & 1.81 & .08 \\
\hline PANAS - negative & $19.85(5.91)$ & $21.27(5.88)$ & -0.98 & .33 \\
\hline
\end{tabular}

FPQ Fear of Pain Questionnaire; $P A N A S$ positive and negative affect; $P T H$ pain threshold; $P T H+11^{\circ} \mathrm{C}$ above pain threshold; $S D$ standard deviation; STAI-T Trait version of the State-Trait Anxiety Inventory.

\section{Manipulation Checks}

The avoidance group indicated that they felt more in control over the intensity of the heat stimulus compared to the control group (Avoidance: $M=50.88, S D=23.60$; Control: $M=20.09, S D=23.67 ; t(64)=6.95, p<.001)$. Groups did not differ in how credible they found the visual feedback (Avoidance $M=67.58, S D=16.45$; Control: $M=68.53, S D=16.29 ; t(64)=-0.23, p=.82)$.

\section{Hypothesis 1: Pain-Related Fear Increases When the Opportunity to Avoid is No Longer Available}

\section{Self-reported pain-related fear}

Figure 2 displays the mean pain-related fear ratings per group measured before (prospective fear; panel a) and after (retrospective fear; panel b) each trial.

Prospective pain-related fear. The RM ANOVA on the prospective pain-related fear ratings revealed a main effect of trial, indicating that the fear ratings changed throughout the experiment, $F(3.75,239.78)=15.47, p<$ $.001, \eta \mathrm{g} 2=.04$. There was no significant main effect of Group, $F(1,64)=0.61, p=.44$. Furthermore, the Group $\times$ Trial interaction effect for prospective pain-related fear failed to reach significance, $F(3.75,239.78)=1.74$, $p=.15$. We tested the hypothesis that prior avoidance behavior would lead to increased pain-related fear for the avoidance group. The planned contrast compared the last trial of the full intensity phase (Trial 3) with the first trial where the avoidance response was no longer available (Trial 7). Consequently, the planned contrast evaluating the change in prospective fear reached significance for the avoidance group, $t(512)=4.32, p<.0001$, and not for the control group, $t(512)=0.55, p=.58$. Furthermore, we tested whether prospective pain-related fear increased as a result of the mere instruction of being able to avoid. We compared the last trial of the full intensity phase
(Trial 3), with the first trial of the intervention phase (just after the instruction was given; Trial 4). The change in prospective pain-related fear was not significant neither for the avoidance group: $t(512)=0.99, p=.32$, nor for the control group: $t(512)=0.88, p=.38$.

Retrospective pain-related fear. The RM ANOVA on the retrospective pain-related fear ratings revealed a main effect of trial, indicating that the retrospective painrelated fear ratings changed throughout the experiment, $F(3.96,253.75)=8.10, p<.0001, \eta \mathrm{g} 2=.02$. There was no significant main effect of Group, $F(1,64)=0.91$, $p=.33$. Furthermore, the Group $\times$ Trial interaction term was not significant, $F(3.96,253.75)=0.68, p=.61$. The planned contrast evaluating the change from the last trial of the full-intensity phase (Trial 3) with the first trial of the test phase (Trial 7) just failed to reach significance for the avoidance group, $t(512)=1.93, p=.054$, and was not significant for the control group, $t(512)=-0.29, p=.77$.

In sum, prior avoidance behavior leads to a significant increase in prospective pain-related fear ratings but failed to reach significance in the retrospective pain-related fear ratings (i.e., after participants experienced the painful stimulus). We did not replicate the finding from our previous study that pain-related fear increased as a result of the mere instruction of being able to avoid [13].

\section{Physiological measure of pain-related fear: startle eyeblink modulation}

Figure 3 displays the mean fear-potentiated startle amplitudes for both groups separately for each trial. The RM ANOVA revealed a significant main effect of Group, $F(1$, $64)=4.33, p<.05, \eta \mathrm{g} 2=.002$. Also, a significant main effect of trial, $F(8,512)=5.68, p<.0001, \eta \mathrm{g} 2=.04$, and Probe, $F(1,64)=239.33, p<.0001, \eta \mathrm{g} 2=.32$, was observed. Furthermore, the Probe $\times$ Trial interaction was significant $F(8,512)=5.11, p<.0001, \eta \mathrm{g} 2=.03$. As a 


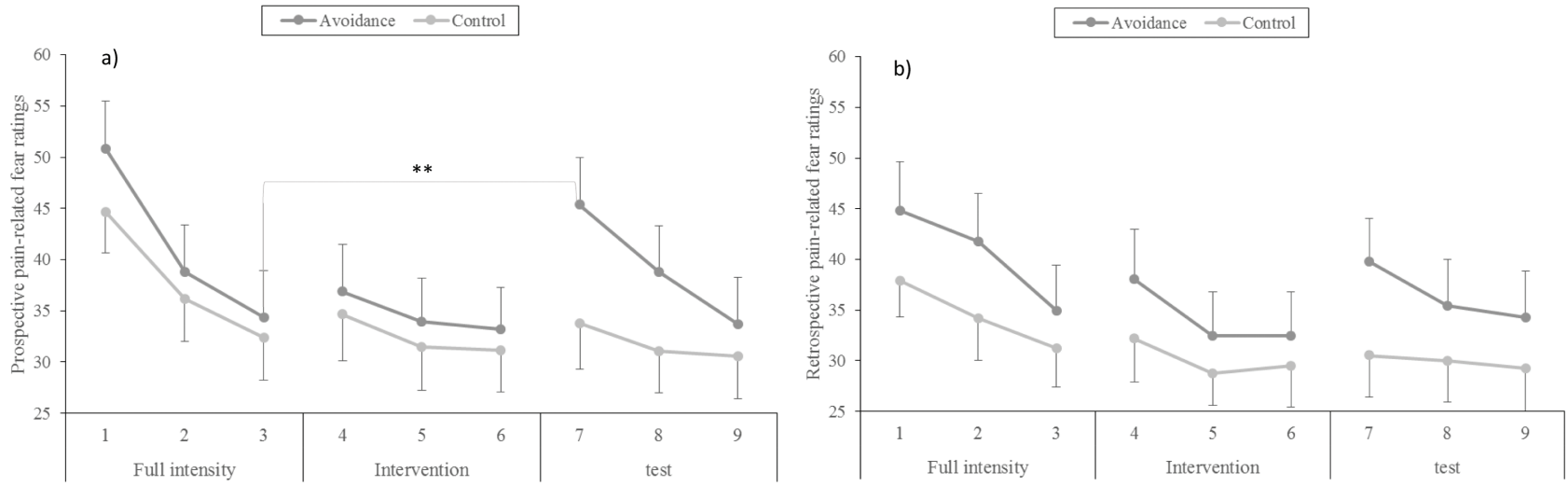

Fig. 2. Prospective (panel a) and retrospective (panel b) pain-related fear ratings with standard error bars for the avoidance group and the control group during the full intensity, intervention, and test phase. ${ }^{* *} p<.01$.

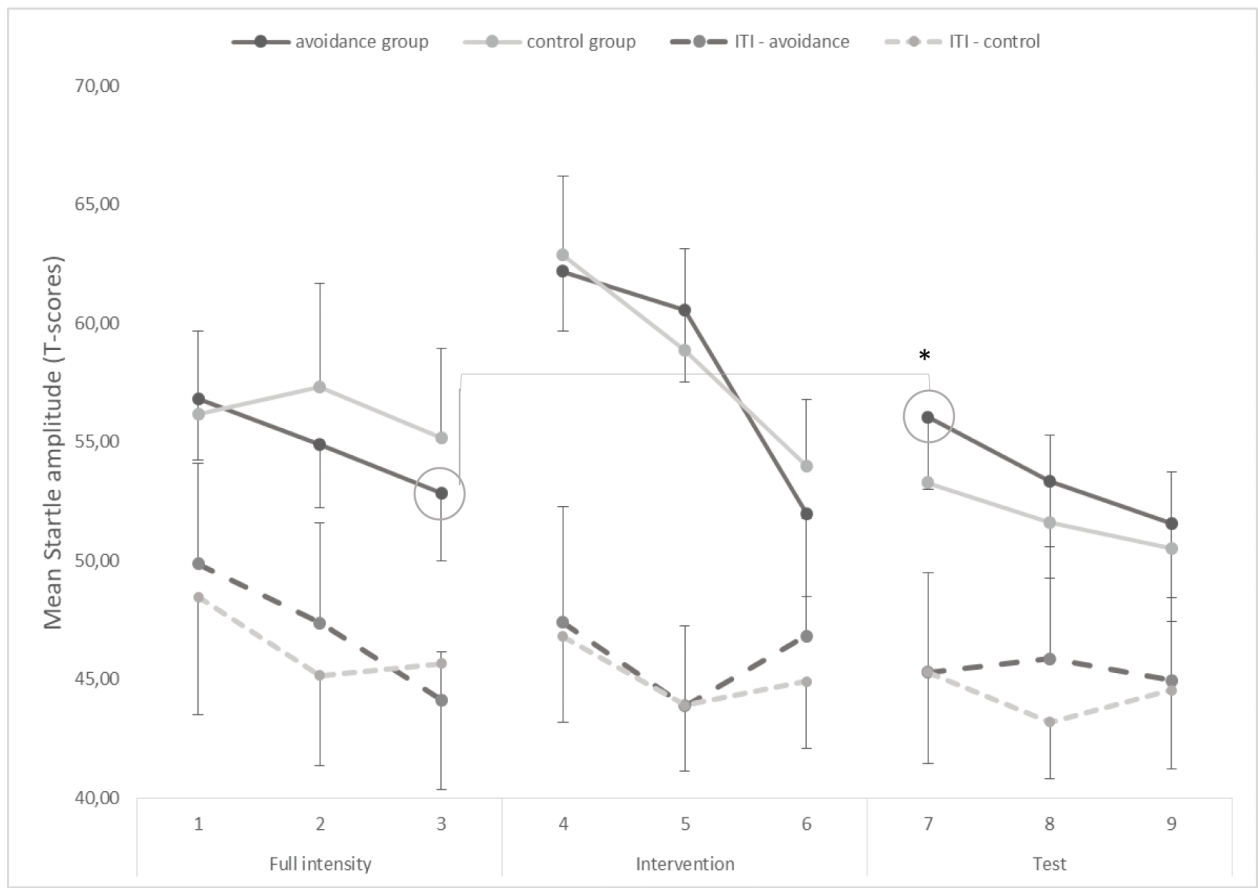

Fig. 3 Mean startle amplitudes for the avoidance group and control group for all trials during stimulation (solid line) and during intertrial interval (ITI; dashed line). The raw scores from the startle measures were converted to Z-scores to account for interindividual differences. For better visualization of the data, the $\mathrm{Z}$-scores were transformed to T-scores to avoid negative values on the $\mathrm{Y}$-axis. ${ }^{*} p<.05$

follow-up to the significant interaction effect, we examined the effect of Probe at each trial level, with a one-way ANOVA. All one-way ANOVAs at trial level were significant. For an overview of the simple main effects, please see Supplementary Table S1. As expected, the startle amplitudes elicited during stimulation were higher than the startle amplitudes during ITI, suggesting that participants were more fearfully aroused during heat stimulation than in absence of the stimulation. The following interactions were not significant: Group $\times$ Trial interaction, $F(8,512)=0.48, p=.87$; Group $\times$ Probe interaction, $F(1,64)=0.0005, p=.98$; Group $\times$ Trial $\times$ Probe interaction, $F(8,512)=0.97, p=.46$. To test our hypothesis that pain-related fear would increase after avoidance behavior, planned contrasts were performed evaluating the changes from the last trial of the full-intensity phase (Trial 3) to the first trial of the test phase (Trial 7). We found a significant increase in the startle amplitude for the avoidance group, $t(1024)=2.26, p=.024$, but not for the control group, $t(1,024)=-1.17, p=.24$. We also observed an increase in startle amplitude during stimulation from the last trial of the full-intensity phase (Trial 3) to the first trial of the intervention phase (Trial 4 ) for the avoidance group, $t(1,024)=3,38, p<.001$, as well as for the control group, $t(1,024)=3.26, p<.001$. The analysis of the mean startle amplitudes on phase level can be found in the Supplementary Material and Supplementary Fig. S1. 

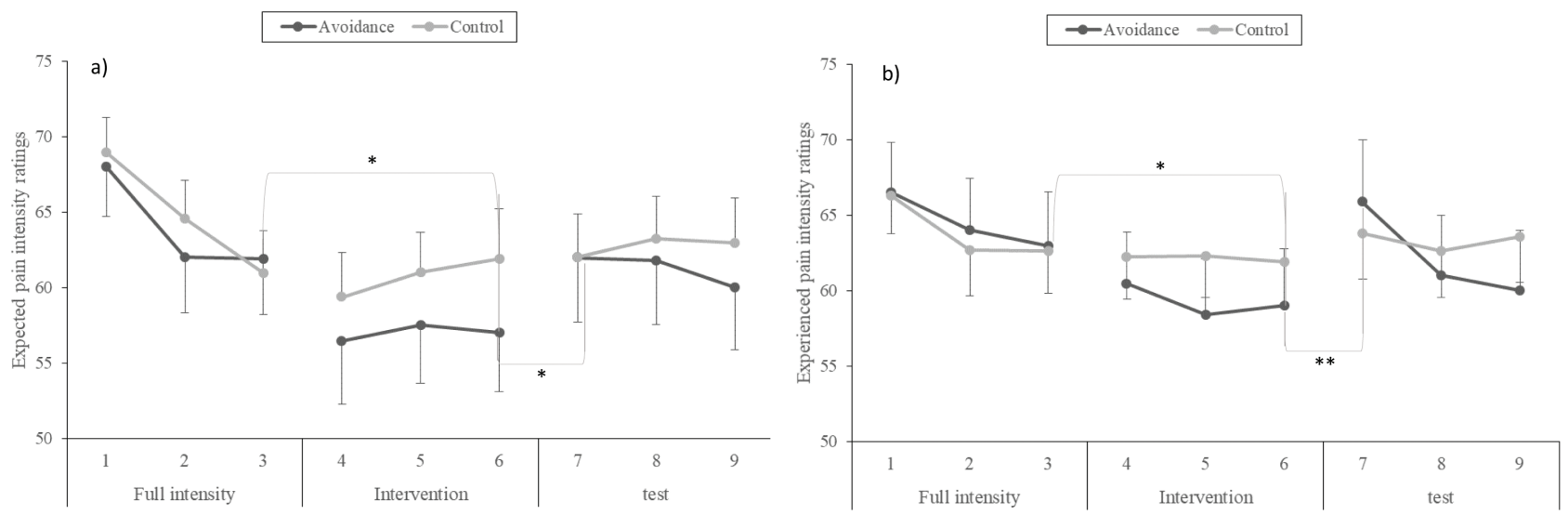

Fig. 4. Expected (panel a) and experienced (panel b) pain intensity ratings with standard error bars for the avoidance group and the control group during the full intensity, intervention, and test phase.

In sum, the mean startle amplitudes seem to corroborate the self-reported increase in prospective pain-related fear after the avoidance response was no longer available (i.e., during the first trial of the test phase). Furthermore, both groups show an increase in startle responses after the avoidance instructions were delivered.

\section{Hypothesis 2: Perceived Avoidance Attenuates Pain Intensity Despite Exposure to Identical Physical Stimulus Intensity}

Figure 4 displays the mean pain intensity ratings per group measured before (expected; panel a) and after (experienced; panel b) each trial.

\section{Expected pain intensity}

Figure 4 (panel a) shows the expected pain intensity ratings for both groups. The RM ANOVA of expected pain intensity showed a significant main effect of trial only, $F(4.65,297.39)=7.75, p<.0001, \mathrm{ng} 2=.02$. The following effects were not significant: main effect of Group, $F(1,64)=0.22, p=.64$; Group $\times$ Trial interaction, $F(4.65,297.39)=0.47, p=.78$. Avoidance group participants expected a lower pain intensity when they performed the avoidance response (intervention phase) compared to the beginning of the experiment where they could not perform an avoidance response (full-intensity phase): last trial of full intensity (Trial 3) versus last trial intervention (Trial 6), $t(512)=-1.95, p<.05$. This was not the case for the control group, $t(512)=-0.14$, $p=.89$. Participants in the avoidance group expected higher pain intensity during the first trial where they could no longer use the avoidance response (test phase; Trial 7) compared to the last trial where the avoidance response was available (intervention phase; Trial 6), $t(512)=1.98, p<.05$. This was not the case for the control group, $t(512)=1.05, p=.30$.

\section{Experienced pain intensity}

Figure 4 (panel b) graphically displays the means of experienced pain intensity ratings for both groups. The RM ANOVA reveals a significant main effect of trial, $F(5.22$, $334.08)=6.75, p<.0001, \eta \mathrm{g} 2=.01$, and just failed to show a significant Group $\times$ Trial interaction, $F(5.22$, $334.08)=1.99, p=.08, \mathrm{\eta g} 2=.003$. The main effect of group was not significant, $F(1,64)=0.07, p=.80$. The planned contrasts evaluating the change in experienced pain intensity during avoiding and not avoiding revealed similar results as the expected pain intensity. Experienced pain intensity significantly decreased for the avoidance group when the avoidance response was available: last trial of full intensity (Trial 3) versus last trial intervention (Trial 6) $t(512)=-2.52, p<.05$, and not for the control group, $t(512)=-0.45, p=.66$. Experienced pain intensity ratings significantly increased for the avoidance group when avoidance behavior was no longer available, $t(512)=4.37, p<.001$, but not for the control group $t(512)=1.20, p=.23$. In sum, perceived avoidance behavior attenuates the expected and experienced pain intensity of physically identical stimuli.

\section{Hypothesis 3: The Threat Appraisal of Pain Mediates the Relationship Between Avoidance Behavior and Pain-Related Fear}

The paths of the mediation model (see Fig. 5) were calculated as follows: (a) the regression of avoidance (group allocation) on $\Delta$ threat, $b=5.38, t(64)=2.56, p<.05$; (b) the regression of $\Delta$ threat on $\Delta$ fear, $b=0.41, t(63)=2.71$, $p<.01$; and finally (c) the regression of avoidance on $\Delta$ fear, controlling for $\Delta$ threat, $b=4.88, t(63)=1.84$, $p=.07$. Bootstrapping the indirect effect of the model gave the following results: indirect effect $=2.18$, standard error $=0.87,95 \% \mathrm{CI}[0.67,4.05]$. Since the $95 \% \mathrm{CI}$ of the indirect effect does not contain 0 , we conclude that mediation has occurred. In other words, an increase in threat 

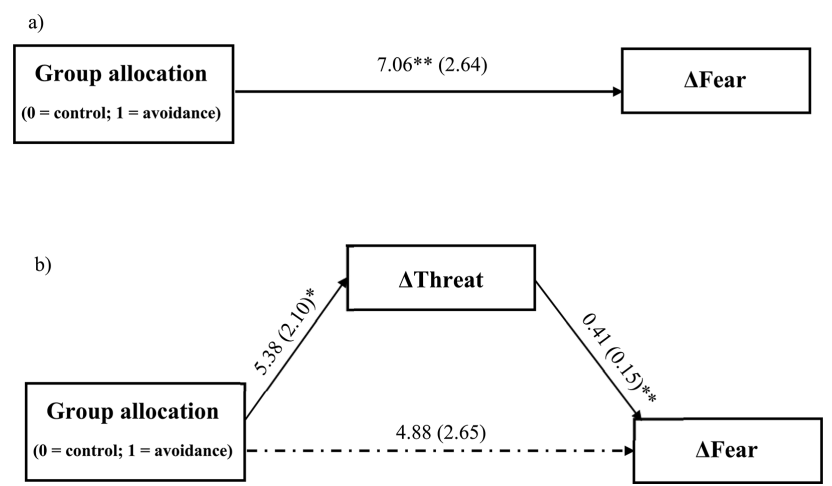

Fig. 5. Panel (a) shows the relation between group allocation and $\Delta$ fear (without mediation). Panel (b) shows $\Delta$ threat as mediator in the relation between group allocation and $\Delta$ fear. All coefficients represent unstandardized regression coefficients. Solid lines represent significant predictions. Dashed lines represent nonsignificant predictions. Values in the brackets represents standard errors. Values of group allocation: $0=$ control group, $1=$ avoidance group. ${ }^{*} p<.05,{ }^{* *} p<.01$.

appraisal, measured as the perceived harmfulness of the painful stimulus, mediated the relationship between the perceived ability to avoid the full pain intensity and changes in pain-related fear. The threat appraisal ratings can be found in Supplementary Fig. S2.

\section{Discussion}

The current study was set up to further test the idea that perceived ability to avoid pain affects pain-related fear. According to modern fear-avoidance models, interpreting pain as threatening (a sign of serious harm) results in pain-related fear, may spur avoidance behavior, and typically maintains chronic pain disability. The initial model proposed a circular but unidirectional relationship between threat appraisal, pain-related fear, and avoidance behavior [7]. However, the unidirectionality of the fear-avoidance relationship has been challenged. Although avoidance of painful stimuli is aimed at reducing the accompanying anticipatory fear and expected pain, previous research has shown that pain avoidance itself can paradoxically increase pain-related fear. In our previous study [13], pain-related fear levels increased after avoiding a painful stimulus, suggesting that the relationship between avoidance behavior and fear may be bidirectional. The current study aimed to replicate these findings and additionally investigate the mediating role of the threat appraisal of pain (i.e., perceived harmfulness) in the avoidance-fear relationship. Compared to the previous study, the current one is notable for the following methodological improvements: (a) pain-related fear and threat appraisals were measured at trial level instead of phase level, enabling the aforementioned mediation analysis; and (b) instructions to avoid the maximal painful heat stimulus when a stop cue is presented are given to both groups instead of to the avoidance group only. This gives us more certainty that the observed increase in pain-related fear in the avoidance group is due to the engagement in avoidance behavior instead of the mere instruction that avoidance is possible.

Findings of the study were the following: we successfully replicated the findings (a) that the prior perceived ability to avoid the painful stimulus increases painrelated fear; (b) that perceived avoidance attenuates pain reports despite exposure to identical stimulus intensity; and additionally found (c) that the threat appraisal of pain mediates the relationship between avoidance behavior and subsequent pain-related fear.

The prospective pain-related fear ratings and psychophysiological data from the eyeblink startle responses support our first hypothesis. Participants of the avoidance group were more afraid when they no longer could perform their avoidance response. These findings are in line with previous research, mainly conducted in the field of anxiety disorders [10-12, 22]. Avoidance behaviors are assumed to play a role in the development and maintenance of excessive fear. Here, we experimentally show that perceived pain avoidance hinders the disconfirmation of pain-related fear and threat appraisals. In this way, misattribution of safety to the avoidance behavior itself occurred, which resulted in decreased pain-related fear scores. That is, pain-related fear was low for the avoidance group during the intervention phase. However, during the crucial test phase, when the avoidance behavior could not be emitted anymore, avoidance group participants reported more pain-related fear, although the stimulus intensity was identical throughout the experiment compared to the control group participants who never avoided.

Our results seem to support our hypothesis that avoidance behavior increases the threat appraisal of a painful stimulus, which then serves as a source of information to further fuel pain-related fear. This model is supported by the idea of ex-consequentia reasoning [23]. The avoidance behavior itself, rather than the painful stimulus, may have led participants to infer danger (e.g., "I avoid, thus there must be danger"). These observations are also in line with findings in the field of anxiety research, where avoidance can be seen as counterproductive and can paradoxically enhance or maintain anxiety/fear and self-reported negative experiences [24-28]. We suggest that people with persistent pain show a similar tendency to attribute the absence of danger (e.g., reinjury) to their avoidance behavior, consequently preventing opportunities to disconfirm threat appraisals [8, 29, 30]. Also, as can be seen from the mediation analysis, perceived threat mediates the relationship between avoidance behavior and pain-related fear. These findings suggest an additional avoidance $->$ threat $\rightarrow>$ pain-related fear loop as an extension to the fear-avoidance model, which may 
act as an additional drive propelling the development of fear-related avoidance and disability levels typical for chronic pain.

Although pain usually is considered an US in itself, it also serves as a cue signaling another US, which is the actual (re)injury or bodily harm. In that context, avoiding painful stimuli (or pain increase) is to prevent the (re) injury to occur. Although avoidance behavior is often described in its passive form, active forms of behavior do also exist, which often are called safety-seeking behaviors, such as deliberately acting in a certain way. In our study, avoidance group participants had the option to engage in active avoidance behavior (i.e., pressing a stop button) and were led to believe that, by doing so, they could avoid pain increase. This paradigm is in line with the fear-avoidance model, even though the avoidance response in question is not refraining from activity (i.e., passive avoidance) but pressing a stop button (i.e., active avoidance or safety-seeking behavior).

Concerning our hypothesis that perceived avoidance behavior attenuates pain intensity reports despite exposure to identical stimulus intensity, we found that the avoidance group participants, but not control group participants, indeed perceived less pain while engaging in avoidance behavior. We propose that the mechanism driving this hypoalgesia could be placebo-inducing expectancies. Specifically, in the experiential phase, participants felt that the stimulus was less intense (i.e., stimulus intensity at the pain threshold level instead of $1^{\circ}$ above the pain threshold level) when the red bar did not reach its maximum. When participants performed the avoidance behavior, again the red bar did not reach its maximum, creating the (placebo) expectation that the stimulus intensity also would not reach its maximum [13, 31]. In the control group, no such differences in pain intensity were found.

In addition to these results, there are other remarkable observations. For the avoidance group, the reduction in pain intensity was not paralleled by reductions in painrelated fear, and this may be due to a bottom effect as the levels of pain-related fear were already quite low. After the instruction to avoid, but prior to actually performing the avoidance response (i.e., pressing the stop button), the eyeblink startle responses increased for both groups. This observed increase in eyeblink startle responses might be a result of changes in attentional processing [32] due to the instruction that avoidance behavior would be available only upon presentation of the stop cue. For example, eyeblink startle responses may have been influenced by preparing to execute an avoidance response (motor preparation). Another potential explanation for the observed increase in the eyeblink startle response is that the instruction to avoid increases attention toward feared stimuli [33], which, in turn, may lead individuals to view themselves more at risk, leading to increased fear levels. As a result, eyeblink startle responses may not be well-suited measures of pain-related fear in this study. Additionally, in our previous study, we found an increase in self-reported pain-related fear as a result of the mere instruction to avoid [13]. Although, in the current study, we did observe an increase in the eyeblink startle responses for both groups, just after the instruction to avoid at the start of the intervention phase, this was not reflected in the self-reported pain-related fear. At this stage, we do not have a plausible explanation for this observation. Furthermore, control participants who were given the same instructions, but never received the opportunity to avoid, displayed significantly lower startle responses during test compared to the start of the experiment, indicating a decrease in fear of the heat stimulus (see Supplementary Material). This was not the case for the avoidance group.

Particular strengths of this study are not only the successful replication of our previous findings [13], corroborating the validity of the paradigm to investigate the effects of pain avoidance on fear and the underlying mechanisms, but also the mediation analysis confirming the mediating role of perceived harmfulness of pain in the relationship between avoidance behavior and fear. Another methodological strength of this study is the operationalization of the avoidance behavior, whereby we created the illusion that participants could avoid the maximum pain by pressing a stop button, although, in reality and unknown to the participants, the intensity and duration of the heat stimulus was independent of the avoidance response. However, there are also some limitations. First, although we mentioned this as one of the strengths of this study, at the same time, the way we operationalized the avoidance can also be seen as a limitation for interpretation of the results in view of the fear-avoidance model of chronic pain because simply pressing a stop button with no associated cost is not ecologically valid. For chronic pain patients, avoidance behavior typically comes with a cost of limitations in daily functioning and, usually, they have more to lose than to gain with their avoidance behavior. However, one could argue that, in real life, both low-cost (e.g., avoiding a simple movement) and high-cost responses (e.g., not participating in valued life activities) are observed in avoidance behaviors of chronic pain patients. We assume the underlying mechanisms of the effects of low- and high-cost responses on pain-related fear and threat to be similar. This study, in which we used a low-cost avoidance response (pressing a stop button) showed effects of this low-cost response on the levels of pain-related fear and threat. Similarly, in real life, we can also observe low-cost avoidance behaviors of chronic pain patients, like carrying pills, just in case pain would increase. These low-cost avoidance behaviors in chronic pain patients could likewise increase pain-related fear and threat [34]. 
Second, we chose to give the avoidance instructions to both groups, which might have created frustration in the control group as they expected the opportunity to avoid the maximal painful stimulation but never received the opportunity to do so. Unfortunately, we did not include a measure of frustration and cannot be sure whether and how this may have affected our primary outcomes. However, the results of the control group were similar to our previous study [13], where no avoidance instructions were given to the control group. Third, this study included pain-free participants. Therefore, one should be cautious in generalizing these findings to individuals with persistent pain. Future studies need to validate these findings using clinical samples. Fourth, the results of this study are based on a very specific situation. Participants in the avoidance group believed that they avoided the maximal pain while the heat stimulus was slowly increasing in temperature. Future studies should consider different experimental pain paradigms to test generality across pain modality. Fifth, although we gave the instruction to press the stop button upon the presentation of a cue to avoid receiving increased pain, we did not record these avoidance responses but, based on observation of the participants during the experiment, most of the participants followed the instruction and pressed the stop button. Finally, please note that, in general, the effect sizes were relatively small and the results were obtained in a heavily controlled environment. Therefore, some caution is warranted in the interpretation of the results.

In conclusion, we corroborated the previous finding that avoidance behavior reduces pain but increases pain-related fear when the avoidance option is lost. Additionally, we experimentally demonstrated that the increase of pain-related fear after previous avoidance behavior is mediated by the threat appraisal of the painful stimulus. These findings suggest a more complicated and interdependent relationship between pain-related fear, avoidance behavior, and threat than the unidirectional relationships originally proposed in the fear-avoidance model. One of the issues according to the fear-avoidance model is the persistence of avoidance behavior, even if this behavior is no longer functional. Indeed, chronic pain patients often continue avoiding movements/activities because they are afraid that these movements amplify their pain or have detrimental consequences. The results of our study indicate that when the avoidance response is no longer available, initially there is a significant increase of pain-related fear, whereas repeated experience of the pain without the avoidance behavior significantly reduces pain-related fear. These data support the use of exposure therapy to reduce pain-related fear in chronic pain patients. As a result, allowing avoidance behaviors in clinical therapy for chronic pain may preserve threat and impede fear reduction [35]. Tackling avoidance behavior to reduce fear seems as important as reducing fear to halt the process of chronicity in individuals with chronic pain. This can be realized by response prevention during repeated exposure to the painful stimuli as was done in the control condition of the current study or by introducing rewards for the decision to perform painful movements [36].

Finally, the current findings can potentially help to further our understanding of how avoidance can maintain pain problems and, therefore, we propose an updated fear-avoidance model with a bidirectional relationship between pain-related fear and avoidance behavior mediated by threat appraisals of pain.

\section{Supplementary Material}

Supplementary material is available at Annals of Behavioral Medicine online.

Acknowledgments This research was supported by the "Asthenes" long-term structural funding Methusalem grant by the Flemish Government, Belgium. A.M. is supported by a Senior Research Fellowship of the Research Foundation Flanders (FWOVlaanderen), Belgium (grant ID: 12E3717N) and by a Vidi grant from the Netherlands Organization for Scientific Research, The Netherlands (grant ID 452-17-002). The authors would like to thank Amine Zerrouk for his assistance in the data collection.

\section{Compliance with Ethical Standards}

Authors' Statement of Conflict of Interest and Adherence to Ethical Standards The authors report no conflict of interest. All procedures in the present study were performed in accordance with the ethical standards of the institutional research committee.

Authors' Contributions C.v.V., A.M., L.V. and J.V. contributed to the concept and design of the study. C.v.V. programmed the experiment, collected the data, and conducted the statistical analyses. C.v.V. wrote the first draft of the manuscript. All authors reviewed and commented on subsequent drafts of the manuscript.

Ethical Approval The Social and Societal Ethics Committee of KU Leuven approved the experimental protocol (Reg \#: G-2017-10-960).

Informed Consent All participants provided written informed consent, which stated that they were allowed to decline participation at any time during the experiment without any consequences.

\section{References}

1. Arntz A, Claassens L. The meaning of pain influences its experienced intensity. Pain. 2004;109:20-25.

2. Meulders A. From fear of movement-related pain and avoidance to chronic pain disability: A state-of-the-art review. Curr Opin Behav Sci. 2019;26:130-136. 
3. Vlaeyen JW, Linton SJ. Fear-avoidance and its consequences in chronic musculoskeletal pain: A state of the art. Pain. 2000;85:317-332.

4. Vlaeyen JW, Linton SJ. Fear-avoidance model of chronic musculoskeletal pain: 12 years on. Pain. 2012;153:1144-1147.

5. Martinez-Calderon J, Flores-Cortes M, Morales-Asencio JM, Luque-Suarez A. Pain-related fear, pain intensity and function in individuals with chronic musculoskeletal pain: A systematic review and meta-analysis. J Pain. 2019;20:1394-1415.

6. Nishi Y, Osumi M, Nobusako S, Takeda K, Morioka S. Avoidance behavioral difference in acquisition and extinction of pain-related fear. Front Behav Neurosci. 2019;13:236.

7. Vlaeyen JW, Kole-Snijders AM, Rotteveel AM, Ruesink R, Heuts PH. The role of fear of movement/(re)injury in pain disability. J Occup Rehabil. 1995;5:235-252.

8. Salkovskis PM. The importance of behaviour in the maintenance of anxiety and panic: A cognitive account. Behav Cogn Psychother. 1991;19:6-19.

9. Lovibond PF, Mitchell CJ, Minard E, Brady A, Menzies RG. Safety behaviours preserve threat beliefs: Protection from extinction of human fear conditioning by an avoidance response. Behav Res Ther. 2009;47:716-720.

10. Engelhard IM, van Uijen SL, van Seters N, Velu N. The effects of safety behavior directed towards a safety cue on perceptions of threat. Behav Ther. 2015;46:604-610.

11. Gangemi A, Mancini F, van den Hout M. Behavior as information: "If I avoid, then there must be a danger". J Behav Ther Exp Psychiatry. 2012;43:1032-1038.

12. van den Hout M, Gangemi A, Mancini F, et al. Behavior as information about threat in anxiety disorders: A comparison of patients with anxiety disorders and non-anxious controls. $J$ Behav Ther Exp Psychiatry. 2014;45:489-495.

13. van Vliet CM, Meulders A, Vancleef LMG, Vlaeyen JWS. The opportunity to avoid pain may paradoxically increase fear. $J$ Pain. 2018;19:1222-1230.

14. Blumenthal TD, Cuthbert BN, Filion DL, Hackley S, Lipp OV, van Boxtel A. Committee report: Guidelines for human startle eyeblink electromyographic studies. Psychophysiology. 2005;42:1-15.

15. Van der Ploeg HM. Validity of the Zelf-BeoordelingsVragenlijst (A Dutch version of the Spielberger State-Trait Anxiety Inventory). Ned Tijdschr Psychol. 1980;35:243-249.

16. van Wijk AJ, Hoogstraten J. Dutch translation of the Fear of Pain Questionnaire: Factor structure, reliability and validity. Eur J Pain. 2006;10:479-486.

17. Engelen U, De Peuter S, Victoir A, Van Diest I, Van Den Bergh O. Further validation of the Positive and Negative Affect Schedule (PANAS) and comparison of two Dutch versions. Gedrag Gezond. 2006;34:61-70.

18. Lonsdorf TB, Menz MM, Andreatta M, et al. Don't fear "fear conditioning": Methodological considerations for the design and analysis of studies on human fear acquisition, extinction, and return of fear. Neurosci Biobehav Rev. 2017;77:247-285.

19. Bakeman R. Recommended effect size statistics for repeated measures designs. Behav Res Methods. 2005;37:379-384.
20. Hayes AF. Introduction to Mediation, Moderation, and Conditional Process Analysis: A Regression-Based Approach. 2nd ed. The Guilford Press; 2018.

21. Meule A. Contemporary understanding of mediation testing. Meta-Psychology 2019;3. doi:10.15626/MP.2018.870.

22. Deacon B, Maack DJ. The effects of safety behaviors on the fear of contamination: An experimental investigation. Behav Res Ther. 2008;46:537-547.

23. Arntz A, Rauner M, van den Hout M. "If I feel anxious, there must be danger": Ex-consequentia reasoning in inferring danger in anxiety disorders. Behav Res Ther. 1995;33:917-925.

24. Campbell-Sills L, Barlow DH, Brown TA, Hofmann SG. Effects of suppression and acceptance on emotional responses of individuals with anxiety and mood disorders. Behav Res Ther. 2006;44:1251-1263.

25. Cioffi D, Holloway J. Delayed costs of suppressed pain. J Pers Soc Psychol. 1993;64:274-282.

26. Dymond S, Schlund MW, Roche B, Whelan R, Richards J, Davies C. Inferred threat and safety: Symbolic generalization of human avoidance learning. Behav Res Ther. 2011;49:614-621.

27. Feldner MT, Zvolensky MJ, Stickle TR, Bonn-Miller MO, LeenFeldner EW. Anxiety sensitivity-physical concerns as a moderator of the emotional consequences of emotion suppression during biological challenge: An experimental test using individual growth curve analysis. Behav Res Ther. 2006;44:249-272.

28. Spira AP, Zvolensky MJ, Eifert GH, Feldner MT. Avoidanceoriented coping as a predictor of panic-related distress: A test using biological challenge. J Anxiety Disord. 2004;18:309-323.

29. Barlow DH. Unraveling the mysteries of anxiety and its disorders from the perspective of emotion theory. Am Psychol. 2000;55:1247-1263.

30. Volders S, Boddez Y, De Peuter S, Meulders A, Vlaeyen JW. Avoidance behavior in chronic pain research: A cold case revisited. Behav Res Ther. 2015;64:31-37.

31. Reicherts P, Gerdes AB, Pauli P, Wieser MJ. Psychological placebo and nocebo effects on pain rely on expectation and previous experience. J Pain. 2016;17:203-214.

32. Valls-Solé J, Valldeoriola F, Tolosa E, Nobbe F. Habituation of the auditory startle reaction is reduced during preparation for execution of a motor task in normal human subjects. Brain Res. 1997;751:155-159.

33. Lavy EH, van den Hout MA. Cognitive avoidance and attentional bias: Causal relationships. Cognit Ther Res. 1994;18:179-191.

34. Vervliet B, Indekeu E. Low-cost avoidance behaviors are resistant to fear extinction in humans. Front Behav Neurosci. 2015;9:351.

35. Meulders A, Van Daele T, Volders S, Vlaeyen JW. The use of safety-seeking behavior in exposure-based treatments for fear and anxiety: Benefit or burden? A meta-analytic review. Clin Psychol Rev. 2016;45:144-156.

36. Claes N, Karos K, Meulders A, Crombez G, Vlaeyen JWS. Competing goals attenuate avoidance behavior in the context of pain. J Pain. 2014;15:1120-1129. 